\title{
Pattern of Upper Respiratory Tract Infections and Physicians' Antibiotic Prescribing Practices in Bahrain
}

\author{
Abiola C. Senok $^{\mathrm{a}} \quad$ Abdulrahman Y. Ismaeel $^{\mathrm{b}}$ Fahad A. Al-Qashar ${ }^{\mathrm{c}}$ \\ Wahid A. Agab ${ }^{c}$ \\ aDepartment of Clinical Sciences, College of Medicine, University of Sharjah, Sharjah, United Arab Emirates; \\ ${ }^{b}$ Department of Microbiology, Immunology and Infectious Diseases, College of Medicine and Medical Sciences, \\ Arabian Gulf University, Manama, and 'Bahrain Defence Force Hospital, West Riffa, Kingdom of Bahrain
}

\section{Key Words}

Upper respiratory tract infections - Antibiotic prescription • Bahrain

\begin{abstract}
Objective: To evaluate the pattern of antibiotic prescriptions for paediatric upper respiratory tract infections (URTI) and determine the associated predictors for such antibiotic use in the Kingdom of Bahrain. Subjects and Methods: From March 2005 to March 2006, demographic data, clinical presentation, investigations and antibiotic prescription for children with URTI $(n=184)$ at the Bahrain Defence Force Hospital was recorded. To assess the factors which influence physician antibiotic prescription for URTI, a cross-sectional survey of doctors was carried out using a pre-tested questionnaire which was administered to paediatricians, general practitioners and emergency room physicians. Results: Antibiotics were given to 95 of the $184(51.6 \%)$ patients, mainly children $<3$ years (40/95). Significant association was demonstrated for antibiotic prescription, age and diagnosis of tonsillitis or acute otitis media $(p<0.05)$. Amoxicillin (37/95) was the most frequently prescribed antibiotic, followed by $\beta$-lactam $/ \beta$-lactamase combination and second-generation
\end{abstract}

\section{KARGER}

Fax +4161306 1234

E-Mail karger@karger.ch

www.karger.com
(C) 2009 S. Karger AG, Basel

$1011-7571 / 09 / 0183-0170 \$ 26.00 / 0$

Accessible online at:

www.karger.com/mpp cephalosporins. Fever, younger age, sore throat and presence of earache increased the likelihood of antibiotic prescription. Data from the cross-sectional survey of doctors revealed that lack of national guidelines, parental pressure and diagnostic uncertainty contributed to antibiotic overuse. Conclusion: Antibiotic overuse for the treatment of paediatric URTI remains a problem in our setting. We suggest the development of national guidelines which are integrated with structured continuing medical education courses, public awareness campaigns and the introduction of rapid streptococcal antigen tests in the outpatient clinic.

Copyright $\odot 2009$ S. Karger AG, Basel

\section{Introduction}

Upper respiratory tract infections (URTI) are among the commonest causes of visits to the doctor in the paediatric age group. The incidence is especially high in the first 7 years of life and children suffer an average of 6 attacks annually. URTI constitutes a major cause of school absenteeism and poses an additional burden on health care delivery. The aetiology is usually viral and, hence, does not necessitate antibiotic prescription. There are 
stringent diagnostic criteria for URTI recommended by the Centers for Disease Control and Prevention (CDC) to help prevent misdiagnosis and inappropriate antibiotic usage $[1,2]$. However, due to parental expectations and attitudes, antibiotic prescription for URTI remains a common practice and occurs in an estimated $50-70 \%$ of patients [3-5]. This overuse of antibiotics reinforces the parental perception that antibiotics are the preferred drugs of choice for treatment of URTI and encourages public demand for them. In addition, it significantly contributes to the emergence of antibiotic-resistant bacteria in the community.

In the Kingdom of Bahrain, antibiotics are not available for purchase over the counter and patients require a prescription from their physician to obtain these drugs. Recent data have shown [6] that approximately one quarter of prescriptions in primary health care settings in the country included antimicrobials, with the majority of these being used for children with respiratory tract infections. A major limitation of that report was that there was no differentiation between prescriptions for URTI and those for lower respiratory tract infections, in which case the antibiotic prescription might have been justified [6]. We have, therefore, conducted this study to clearly assess the pattern of antibiotic prescriptions in paediatric URTI in the Kingdom of Bahrain and to determine the predictors for such antibiotic use.

\section{Subjects and Methods}

\section{Setting}

The Kingdom of Bahrain is a group of small islands located in the Arabian Gulf with a population of approximately 650,000. The study was conducted at the paediatric outpatient clinics of the Bahrain Defence Force Hospital from March 2005 to March 2006. This 400-bed hospital covers almost all specialities and is the second largest in the country. The outpatient clinic covers both specialized and general outpatient clinics. The attending doctors at the paediatric outpatient clinic include consultants, residents and interns. Data were collected for patients with a diagnosis of URTI. Patients with immunocompromised status, lower respiratory tract infections, chronic respiratory tract infections or with laboratory-proven infections were excluded. Those with symptoms suggestive of bacterial infections in other parts of the body (e.g. skin infections, gastrointestinal infections) were also excluded.

\section{Subjects}

During the study period, 184 children diagnosed with URTI were enrolled into the study. 116 were boys and 68 were girls, and their ages ranged from 1 month to 14 years. Cough and fever were the 2 major presenting symptoms, occurring in 120 children. Rhinorrhoea was present in 112 children, but this was reported as
Table 1. Diagnosis of URTI and antibiotic prescription

\begin{tabular}{lll}
\hline Diagnosis & $\begin{array}{l}\text { Patients } \\
\text { diagnosed }\end{array}$ & $\begin{array}{l}\text { Patients prescribed } \\
\text { antibiotics }\end{array}$ \\
\hline Common cold & 50 & $6(13 \%)$ \\
Bronchiolitis & 25 & $8(32 \%)$ \\
Pharyngitis & 48 & $29(60.4 \%)$ \\
Acute otitis media & 26 & $21(80.7 \%)$ \\
Tonsillitis & 35 & $31(88.5 \%)$ \\
\hline Total & 184 & $95(51.6 \%)$ \\
\hline
\end{tabular}

being purulent in only 11 children. The mean duration of symptoms was $2.4 \pm 1.7$ days.

Data were collected on patient demographics, presenting symptoms, duration of illness and physical examination findings from the case files of eligible patients with URTI, using a data collection form. The name of the attending physician was noted, any request for laboratory investigation and type of investigations were also recorded. The medications prescribed for the patients, the dosage and duration were also noted.

\section{Survey of Doctors}

To assess the factors which influenced physicians' decisions to prescribe antibiotics for URTI, we conducted a cross-sectional survey of 26 doctors (12 paediatricians, 4 general practitioners and 10 emergency medicine doctors) using a pre-tested questionnaire. The questions enquired about which URTI they were likely to prescribe antibiotics for and the factors which influenced this decision, their preferred antibiotics and the factors influencing their choice. They were also asked to identify the most likely reasons for antibiotic overuse in URTI in our setting.

\section{Statistics}

Statistical analysis ( $\chi^{2}$ test and multiple regression) was done using SigmaStat version 3.5 (Systat Software Inc., San Jose, Calif., USA) and $\mathrm{p}<0.05$ was considered statistically significant.

\section{Results}

\section{Symptoms and Signs}

Common cold (fever with cough and runny nose) was the most common diagnosis, reported in 60 (33\%) children, and 25 were diagnosed with acute bronchitis. The diagnosis of pharyngitis and tonsillitis was associated with children who had fever accompanied by inflamed oropharynx. Compared to those diagnosed with pharyngitis, children who were determined to have tonsillitis demonstrated a trend of higher temperatures and associated complaint of sore throat, and 5 had lymphadenopathy. There was no record of enlarged tonsils. The diagno- 
Table 2. Distribution of antibiotics prescribed for different types of URTI

\begin{tabular}{|c|c|c|c|c|c|c|c|c|}
\hline \multirow[t]{2}{*}{ Diagnosis } & \multicolumn{7}{|l|}{ Antibiotics } & \multirow[t]{2}{*}{ Total } \\
\hline & amoxicillin & $\begin{array}{l}\text { amoxicillin/ } \\
\text { clavulanate }\end{array}$ & cefaclor & cefuroxime & cefprozil & erythromycin & azithromycin & \\
\hline Common cold & 2 & - & 3 & 1 & - & - & - & 6 \\
\hline Bronchiolitis & 4 & 4 & - & - & - & - & - & 8 \\
\hline Pharyngitis & 15 & 5 & 3 & - & 1 & 3 & 2 & 29 \\
\hline Tonsillitis & 8 & 13 & 6 & - & 2 & 1 & 1 & 31 \\
\hline Acute otitis media & 8 & 9 & 3 & - & - & - & 1 & 21 \\
\hline Total & 37 & 31 & 15 & 1 & 3 & 4 & 4 & 95 \\
\hline
\end{tabular}

sis of otitis media $(n=26)$ was associated with inflamed/ hyperaemic tympanic membrane, but none of the children had ear discharge.

\section{Laboratory Investigations and Antibiotic Prescription}

All decisions for antibiotic use were made on empirical grounds and laboratory investigations (throat swabs for streptococcal infection and full blood counts) were requested only for 10 children (8 for tonsillitis, 2 for pharyngitis). Over half of the children [95/184 (52\%)] received prescriptions for antibiotics (table 1). In rank order, antibiotic prescription was highest $(>80 \%)$ for children diagnosed with tonsillitis and acute otitis media and lowest for those with a diagnosis of common cold. All 6 children with common cold who were given antibiotics had purulent rhinorrhoea. Diagnosis of tonsillitis and acute otitis media were significantly associated with antibiotic prescription $(\mathrm{p}<0.05)$. There was no association between the duration of symptoms and antibiotic prescription. A large proportion of the antibiotic prescriptions were made for children under the age of 3 years [40/95 (42.1\%)] and there was a significant association between age and antibiotic use $(p<0.05)$. Multiple linear regression analysis controlling for variations in time of presentation, duration of symptoms and the attending physician showed that independent association persisted for antibiotic prescription in children under the age of 3 years $(\mathrm{p}<$ $0.001)$.

The antibiotics prescribed were (in descending rank order): amoxicillin, amoxicillin/clavulanate cefaclor, erythromycin, cefprozil and cefuroxime for 5-7 days, and azithromycin for 3 days (table 2). No patient was prescribed more than 1 antibiotic. The non-antimicrobial medications prescribed were decongestants, cough suppressants and antipyretics. There was no association be- tween the qualifications of the attending doctor and antibiotic prescription.

\section{Survey of Doctors}

All 26 doctors were least likely to prescribe antibiotics for common cold and most likely to prescribe for tonsillitis and acute otitis media. The factors identified as influencing the likelihood of antibiotic prescription were (in descending rank order): fever, younger age, sore throat and presence of earache. However, compared to general practitioners $[n=3 / 10(30 \%)]$, a significantly higher number of paediatricians and emergency medicine physicians $[\mathrm{n}=10 / 16(69 \%)]$ identified purulent rhinorrhoea as an influencing factor for antibiotic use $(\mathrm{p}<0.05)$. The 2 most common drugs of choice identified were amoxicillin and amoxicillin/clavulanate. Penicillin V was the least popular drug of choice, with only 2 (7.6\%) doctors indicating that they would prescribe this antibiotic for patients with URTI. Availability, spectrum of activity and knowledge of local resistance patterns were the top 3 factors that influenced the choice of antibiotic. The top 3 contributory reasons for antibiotic over prescribing, as identified in the survey, were lack of national guidelines, parental pressure or demand and diagnostic uncertainty.

\section{Discussion}

The data presented here represent the first report on the pattern of antibiotic prescription for paediatric cases of URTI in Bahrain. We identified the factors which influenced antibiotic prescribing habits for URTI. The findings show that $52 \%$ of patients received antibiotics and indicate that there is significant overuse of antimicrobials in the management of URTI in our setting. URTI 
is usually of viral aetiology and represents one of the most common reasons for visits to primary-care facilities [7]. However, several studies show that many physicians continue to prescribe antibiotic treatment for URTI, thus contributing to the continued development of antibiotic resistance [8-13]. In a recently published article, antimicrobials were described as the fourth most commonly prescribed drugs in primary health-care facilities in Bahrain, with the majority of these being prescribed to children [6]. It has been suggested that faced with an ill-looking, febrile child (in particular younger children) and anxious parents, physicians are more likely to prescribe antibiotics rather than offer symptomatic treatment [3]. Indeed, our findings demonstrated that clinical features suggestive of a sick child, including higher temperature, sore throat, purulent rhinorrhoea and earache, as well as younger age ( $<3$ years) were all associated with antibiotic prescription. This was confirmed by the finding from the cross-sectional survey of doctors which showed that these are the same factors which would influence their decisions to prescribe antibiotics.

Thus, when faced with diagnostic uncertainty physicians are likely to err on the side of caution, and the current lack of national guidelines was identified as a driving force for overprescription of antibiotics. There is an urgent need for the development of evidence-based antimicrobial prescribing guidelines endorsed by relevant national/regional medical organizations. This should be widely disseminated to health-care providers and pharmaceutical companies. Such guidelines will drive rational antimicrobial prescribing and reduce antibiotic overuse.

It has been reported that $30-50 \%$ of parents expect or express a wish for their child with URTI to receive antibiotic treatment when seen by the attending doctor $[4$, 14-16]. This parental expectation has been described as a significant predictor of physician antibiotic prescribing for conditions of viral aetiology. In settings such as ours where antibiotics are prescription-only drugs, there is the tendency towards parental expectation for antibiotics when they attend the primary health-care facility and, indeed, some parents will request antibiotics [3]. This parental expectation and pressure for antibiotics was identified as an influencing factor for antibiotic prescription for children with URTI. Thus, there is a need for physicians, opinion leaders and medical organizations to play a role in educating the public on the dangers of antibiotic misuse via print and electronic media. In addition, we suggest further work to determine the degree of parental pressure for antibiotics which exists in our setting and identify the driving factors behind this expectation.

Antibiotic Prescription for Upper

Respiratory Tract Infections
The greatest number of prescriptions were for tonsillitis and acute otitis media and the fewest were for common cold. While this pattern is similar to what has been described in other settings, it is of interest that the proportion of patients with common cold who received antibiotics (13\%) in this study is still relatively low compared to findings in other reports $[9,12,13,17]$. Most sore throats are of viral aetiology and the only common cause of sore throat warranting antibiotics is group A $\beta$-haemolytic streptococci. The American Academy of Pediatrics, the CDC and the Infectious Disease Society of America recommend performance of a group A $\beta$-haemolytic streptococci test prior to treatment of sore throat with antibiotics in children $[1,10,18]$. Whilst antibiotic therapy was particularly high (60-80\%) among children diagnosed with pharyngitis and tonsillitis, throat swabs were obtained for investigation in only 10 patients. Thus, to expedite the diagnosis of URTI of bacterial aetiology and thus minimize the empirical use of antibiotics in children, we suggest that rapid streptococcal antigen testing should be introduced in primary-care settings.

The CDC recommends penicillin as the drug of choice for children with sore throat caused by group A $\beta$-haemolytic streptococci, with amoxicillin, first-generation cephalosporins and erythromycin listed as suitable alternatives. Surprisingly, only 2 doctors indicated that they were likely to prescribe penicillin $\mathrm{V}$, which has been proven to be cheap and safe, with a targeted spectrum and be effective for group A $\beta$-haemolytic streptococci. It was found to be the most commonly prescribed antibiotic for patients with URTI in a survey of doctors in 3 Scandinavian countries and ranked second to macrolides in Germany [19-21]. However, this drug was not prescribed for any patient during the study period and the top 3 antibiotics prescribed were amoxicillin, amoxicillin/clavulanate and cefaclor. This corresponded with findings from the survey of doctors which showed that amoxicillin and amoxicillin/clavulanate were the antibiotics they were most likely to prescribe for paediatric URTI when deemed necessary. This choice of antibiotic is in keeping with reported physician prescribing habits in other studies $[9,10$, $12,17]$. Amoxicillin, which was the antibiotic most frequently prescribed in this report [38.9\% (37/95)], is a recommended drug as per the international guidelines; however, there remains a need to address the continued prescription of the $\beta$-lactam $/ \beta$-lactamase combination, second-generation cephalosporins and macrolides, which are not in keeping with recommended guidelines. The diversity of antibiotic types likely to be prescribed by doctors in our setting was limited, with only 7 antibiotic 
types being prescribed to patients during the study. This is in contrast with other studies where between 10 and 25 types of antibiotics have been identified to be in use for paediatric URTI $[9,10,12,17]$. This finding is unlikely to be related to issues of availability or cost as free health care, including dispensing of essential drugs, is available to both citizens and residents in the country. Indeed, we speculate that knowledge of local resistance patterns, which was one of the influencing factors for antibiotic choice, might explain this observation. However, there is a need for clear documentation of these local or regional resistance patterns to provide a more objective guide for clinicians in their antibiotic selection.

\section{Conclusion}

Antibiotic overuse for the treatment of paediatric URTI remains a problem in our setting. We recommend a number of multidimensional initiatives including development of national guidelines based on local knowledge of clinical presentation and antimicrobial resistance patterns, the integration of these guidelines into structured continuing medical education courses, public health awareness campaigns actively supported by opinion leaders in the medical community as well as the introduction of rapid streptococcal antigen tests in outpatient clinics to improve diagnostic accuracy.

\section{References}

1 Dowell SF, Schwartz B, Phillips WR: Appropriate use of antibiotics for URIs in children. 2. Cough, pharyngitis and the common cold: the pediatric URI consensus team. Am Fam Physician 1998;58:1335-1342.

$>2$ Dowell SF, Schwartz B, Phillips WR: Appropriate use of antibiotics for URIs in children. 1. Otitis media and acute sinusitis: the pediatric URI consensus team. Am Fam Physician 1998;58:1113-1118.

$\checkmark 3$ Pichichero ME: Understanding antibiotic overuse for respiratory tract infections in children. Pediatrics 1999;104:1384-1388.

$\checkmark 4$ Shlomo V, Adi R, Eliezer K: The knowledge and expectations of parents about the role of antibiotic treatment in upper respiratory tract infection: a survey among parents attending the primary physician with their sick child. BMC Fam Pract 2003;4:20.

$\checkmark 5$ Watson RL, Dowell SF, Jayaraman M, Keyserling H, Kolczak M, Schwartz B: Antimicrobial use in pediatric upper respiratory infections: reported practice, actual practice and parent beliefs. Pediatrics 1999;104:12511257.

$\checkmark 6$ Al Khaja KA, Sequeira RP, Damanhori AH, Ismaeel AY, Handu SS: Antimicrobial prescribing trends in primary care: implications for health policy in Bahrain. Pharmacoepidemiol Drug Saf 2008;7:389-396.

$>7$ Mlynarczyk G, Mlynarczyk A, Jeljaszewicz J: Epidemiological aspects of antibiotic resistance in respiratory pathogens. Int J Antimicrob Agents 2001;18:497-502.
$>8$ Harnden A, Perera R, Brueggemann AB, Mayon-White R, Crook DW, Thomson A, Mant D: Respiratory infections for which general practitioners consider prescribing an antibiotic: a prospective study. Arch Dis Child 2007;92:594-597.

$>9$ Leblebicioglu H, Canbaz S, Peksen Y, Gunaydin M: Physicians' antibiotic prescribing habits for upper respiratory tract infections in Turkey. J Chemother 2002;14:181-184.

10 Linder JA, Bates DW, Lee GM, Finkelstein JA: Antibiotic treatment of children with sore throat. JAMA 2005;294:2315-2322.

11 McCaig LF, Besser RE, Hughes JM: Trends in antimicrobial prescribing rates for children and adolescents. JAMA 2002;287:30963102.

12 Mohan S, Dharamraj K, Dindial R, Mathur D, Parmasad V, Ramdhanie J, Matthew J, Pinto Pereira LM: Physician behaviour for antimicrobial prescribing for paediatric upper respiratory tract infections: a survey in general practice in Trinidad, West Indies. Ann Clin Microbiol Antimicrob 2004;3:11.

13 Nyquist AC, Gonzales R, Steiner JF, Sande MA: Antibiotic prescribing for children with colds, upper respiratory tract infections, and bronchitis. JAMA 1998;279:875-877.

14 Braun BL, Fowles JB: Characteristics and experiences of parents and adults who want antibiotics for cold symptoms. Arch Fam Med 2000;9:589-595.
15 Mangione-Smith R, McGlynn EA, Elliott MN, McDonald L, Franz CE, Kravitz RL: Parent expectations for antibiotics, physician-parent communication, and satisfaction. Arch Pediatr Adolesc Med 2001;155: 800-806.

16 Palmer DA, Bauchner H: Parents' and physicians' views on antibiotics. Pediatrics 1997; 99:E6.

17 Chlabicz S, Malgorzata-Oltarzewska A, Pytel-Krolczuk B: Respiratory tract infections: diagnosis and use of antibiotics by family physicians in north-eastern Poland. Int J Antimicrob Agents 2004;23:446-450.

18 Bisno AL: Acute pharyngitis. N Engl J Med 2001;344:205-211.

19 Andre M, Vernby A, Odenholt I, Lundborg CS, Axelsson I, Eriksson M, Runehagen A, Schwan A, Molstad S: Diagnosis-prescribing surveys in 2000, 2002 and 2005 in Swedish general practice: consultations, diagnosis, diagnostics and treatment choices. Scand J Infect Dis 2008;40:648-654.

20 Odenholt I, Bylander-Groth A, FrimodtMoller N, Rokstad KS, Molstad S: Differences in antibiotic prescribing patterns between general practitioners in Scandinavia: a questionnaire study. Scand J Infect Dis 2002;34: 602-609.

21 Schindler C, Krappweis J, Morgenstern I, Kirch W: Prescriptions of systemic antibiotics for children in Germany aged between 0 and 6 years. Pharmacoepidemiol Drug Saf 2003; 12:113-120. 